\section{PRESCRIÇÕES DE MEDICAMENTOS EM UNIDADE DE CUIDADOS paliativos de um hospital universitário de Porto Alegre}

\author{
DRUG PRESCRIPTIONS IN A PALLIATIVE CARE UNIT OF A \\ unIVERSity hospital in Porto Alegre
}

\author{
Ester Duk Schwarz¹, Stéphanie Oliveira Baggio², Denise Bueno ${ }^{2,3}$
}

\section{RESUMO}

Introdução: Em menos de 40 anos, o Brasil passou de um perfil de mortalidade típico de uma população jovem para um perfil caracterizado por enfermidades complexas e mais onerosas, próprias das faixas etárias mais avançadas. O controle dos sintomas de pacientes em cuidados paliativos pode melhorar a qualidade de vida desses indivíduos e até mesmo de suas famílias. A utilização de medicamentos adequados para a terapia desses pacientes é fundamental para o sucesso do tratamento, que nesse caso é o controle adequado dos sintomas. O objetivo principal do trabalho foi descrever o perfil de utilização de medicamentos em pacientes em cuidados paliativos internados no Hospital de Clínicas de Porto Alegre (HCPA).

Métodos: O presente estudo descritivo, exploratório tem por objetivo determinar o perfil de uma amostra de 30 pacientes internados na unidade de cuidados paliativos do HCPA com enfoque na utilização de medicamentos e também nas características demográficas e clinicas e o desfecho clínico dos pacientes.

Resultados: As prescrições levantadas totalizaram 76 medicamentos diferentes; dentre estes, os mais prescritos foram morfina, dipirona, lactulose, heparina, metoclopramida, paracetamol, cloreto de sódio, ondansetron, ipratróprio, dexametasona e omeprazol. Com a possibilidade de acúmulo de patologias crônicas, a polifarmácia é inevitável. A avaliação do uso dos fármacos torna-se de grande importância para que se garanta a segurança do paciente em cuidado paliativo e o uso racional de medicamentos.

Conclusão: A construção metodológica deste estudo possibilitou descrever o perfil da população de interesse, além de gerar conhecimento sobre os medicamentos utilizados na unidade de cuidados paliativos e sobre variáveis demográficas e clínicas de pacientes internados nessa unidade.

Palavras-chave: Cuidados paliativos; assistência farmacêutica; preparações farmacêuticas

\section{ABSTRACT}

Introduction: In less than 40 years, Brazil changed from a mortality profile typical of a young population to one characterized by complex and more costly diseases, typical of older ages. The control of the symptoms of patients in palliative care can improve the quality of life of these individuals and even of their families. The use of appropriate drugs for the treatment of these patients is critical to therapeutic success, which in this case is adequate control of symptoms. The main objective of this study was to describe drug use profile in patients under palliative care admitted to Hospital de Clínicas de Porto Alegre (HCPA).
Clin Biomed Res. 2016;36(1):27-36

1 Faculdade de Farmácia, Universidade Federal do Rio Grande do Sul (UFRGS). Porto Alegre, RS, Brasil.

2 Programa de Pós-graduação em Assistência Farmacêutica, Faculdade de Farmácia, Universidade Federal do Rio Grande do Sul (UFRGS). Porto Alegre, RS, Brasil.

3 Departamento de Produção e Controle de Medicamentos, Faculdade de Farmácia, Universidade Federal do Rio Grande do Sul (UFRGS). Porto Alegre, RS, Brasil.

Autor correspondente: Denise Bueno denise.bueno@ufrgs.br Departamento de Produção e Controle de Medicamentos, Faculdade de Farmácia, Universidade Federal do Rio Grande do Sul (UFRGS) Avenida Ipiranga, 2752. 90610-000, Porto Alegre, RS, Brasil. 
Methods: The present descriptive, exploratory study aimed to determine the profile of a sample of 30 patients admitted to the palliate care unit of HCPA focusing on drug use and also on patients' demographic and clinical characteristics and clinical outcomes.

Results: The prescriptions included a total of 76 different drugs, most commonly prescribed of which were morphine, dipyrone, lactulose, heparin, metoclopramide, acetaminophen, sodium chloride, ondansetron, ipratropium, dexamethasone and omeprazole. With the possibility of accumulation of chronic pathologies, polypharmacy is inevitable. The evaluation of drug use becomes very important to ensure patient safety in palliative care and the rational use of drugs.

Conclusions: The methodological construction of this study made it possible to describe profile of the population of interest and provided knowledge about the drugs used in the palliative care unit and about demographic and clinical variables of patients admitted to this unit.

Keywords: Palliative care; pharmaceutical services; pharmaceutical preparations

Há uma tendência mundial para o envelhecimento populacional. Diminuição da fertilidade e aumento significativo da expectativa de vida no último século levaram a uma mudança das causas de doenças e de morte. No início do século XX, a maior ameaça à saúde eram doenças infecciosas e parasitárias que geralmente acometiam crianças. Atualmente são as doenças não transmissíveis, as quais afetam adultos e idosos, que têm maior impacto na saúde mundial. Mudanças na estrutura familiar e envelhecimento populacional significam uma maior população idosa com pouco apoio familiar, que deve ser acolhida por uma sociedade que ainda precisa aprender a lidar com esse novo problema ${ }^{1}$.

Estudos do Instituto Brasileiro de Geografia e Estatística (IBGE) mostram que, entre 1901 e 2010, a população brasileira passou de 17,4 para 190,8 milhões de pessoas, $84 \%$ das quais vivem em área urbana; além disso, a expectativa de vida do brasileiro subiu de 33,4 anos em 1910 para 73,17 anos em 2009 (77,0 anos para mulheres e 69,4 para homens). A população de brasileiros acima de 65 anos passou de 4,9\% em 1995 para 6,1\% em 2005 e 7,4\% em 2010. Em menos de 40 anos, o Brasil passou de um perfil de mortalidade típico de uma população jovem para um perfil caracterizado por enfermidades complexas e mais onerosas, próprias das faixas etárias mais avançadas. Os grandes centros urbanos, embora já apresentem um perfil demográfico semelhante ao dos países mais desenvolvidos, ainda não dispõem de uma infraestrutura de serviços que dê conta das demandas decorrentes das transformações demográficas vigentes ${ }^{2,3}$. Junto com o prolongamento da vida, os profissionais de saúde começaram a perceber que, mesmo no caso de doenças sem cura, há uma possibilidade de atendimento, com ênfase na qualidade de vida e nos cuidados aos pacientes, por meio de assistência interdisciplinar, e na abordagem aos familiares - os cuidados paliativos $(C P)^{4,5}$.
Com a evolução da forma de vida e morte na sociedade, a Organização Mundial de Saúde (OMS) introduz em 1990 o conceito de CP, atualizado em 2002:

Cuidados Paliativos consistem na assistência promovida por uma equipe multidisciplinar, que objetiva a melhoria da qualidade de vida do paciente e seus familiares, diante de uma doença que ameace a vida, por meio da prevenção e alívio do sofrimento, da identificação precoce, avaliação impecável e tratamento de dor e demais sintomas físicos, sociais, psicológicos e espirituais $(\mathrm{WHO})^{6}$.

\section{Os princípios preconizados são:}

[...] fornecer alívio para dor e outros sintomas estressantes como fadiga, anorexia, dispneia e outras emergências oncológicas; reafirmar a vida e a morte como processos naturais; integrar os aspectos psicológicos, sociais e espirituais ao aspecto clínico de cuidado do paciente; não apressar ou adiar a morte; oferecer um sistema de apoio para ajudar a família a lidar com a doença do paciente, em seu próprio ambiente; oferecer um sistema de suporte para ajudar os pacientes a viverem o mais ativamente possível até sua morte; usar uma abordagem interdisciplinar para acessar necessidades clínicas e psicossociais dos pacientes e suas famílias, incluindo aconselhamento e suporte ao luto $(\mathrm{WHO})^{6}$.

$\mathrm{Na}$ Europa se desenvolve o modelo de CP a partir de 1960, inicialmente voltado ao cuidado de pacientes oncológicos. Órgãos internacionais começam o debate sobre essa temática em 1980. $\mathrm{Na}$ década de 90 , os primeiros estudos europeus com dados comparativos de CP começam a ser publicados, possibilitando a percepção de que os CP apresentam diferenças relacionadas a particularidades do sistema de saúde, aspectos culturais e formação profissional?.

A Rede de Pesquisa da Associação Europeia de Cuidados Paliativos (European Association of Palliative Care Research Network), fundada em 
1988, reconhece que as redes de cuidado em saúde são essenciais para a pesquisa sobre CP. Grupos de trabalho foram criados para elaborar diretrizes clínicas baseadas nas evidências disponíveis. Essas diretrizes geralmente têm sido úteis na identificação de lacunas na base de evidências e nas áreas prioritárias de investigação ${ }^{8}$.

No Brasil, iniciativas isoladas e discussões a respeito dos CP são encontradas desde os anos 1970. O movimento dos CP, como filosofia de trabalho, começou no início na década de 1980, com os primeiros serviços instituídos no Rio Grande do Sul, em São Paulo e em Santa Catarina. Foi nos anos 1990 que começaram a aparecer os primeiros serviços organizados, ainda de forma experimental. No Rio de Janeiro, a partir de 1998, CP são prestados nas modalidades de atendimento ambulatorial, internação hospitalar e assistência domiciliar, levando esse estado a tornar-se referência nacional no ensino e capacitação de profissionais para o atendimento em CP. Em 2005, um grupo de médicos de diferentes áreas de atuação, como Geriatria, Pediatria, Oncologia e Medicina de Família, fundou a Academia Nacional de Cuidados Paliativos, cujo mote principal foi o esclarecimento, a divulgação e a promoção dos $\mathrm{CP}$ no Brasil. Esse movimento foi crescendo e se ampliando, levando o Conselho Federal de Medicina a criar a Câmara Técnica sobre a Terminalidade da Vida. Em 2006, foi criada a Resolução n 1.805/2006, então polêmica e ao mesmo tempo um marco em relação à legislação dos $\mathrm{CP}$ no Brasil, contribuindo decisivamente para a aprovação da medicina paliativa como especialidade médica em dezembro de $2011^{9}$.

O alívio do sofrimento é universalmente reconhecido como um objetivo fundamental da assistência em saúde. O controle dos sintomas de pacientes em CP pode melhorar a qualidade de vida desses indivíduos e até mesmo de suas famílias. A utilização de medicamentos de maneira adequada para a terapia desses pacientes é fundamental para o sucesso do tratamento, que nesse caso é o controle adequado dos sintomas.

A composição da equipe multiprofissional clássica de CP está centrada nos profissionais que lidam diretamente com o paciente (médicos, enfermeiros, técnicos de enfermagem, psicólogos e assistentes sociais), o que acaba refletindo na quantidade de literatura disponível sobre o assunto. Segundo Hermes \& Lamarca, a categoria que mais publica sobre a temática é a enfermagem, devido à própria formação baseada na arte de cuidar. Os aspectos mais comuns levantados nos artigos foram: humanização do atendimento, despreparo profissional em relação à morte, eutanásia, distanásia, ortotanásia, mistanásia e currículos que carecem de disciplinas voltadas para a tanatologia ${ }^{4}$.
Estudos de utilização de medicamentos permitem avaliar como os medicamentos são utilizados e identificar problemas relacionados à sua utilização, podendo possibilitar ações educativas para a equipe e auxiliar no planejamento de ações futuras. São poucos os estudos de utilização de medicamentos em pacientes sob CP; portanto, o maior conhecimento sobre esses aspectos pode auxiliar os profissionais de saúde atuantes nessa área. O objetivo principal deste estudo foi analisar os medicamentos utilizados em pacientes sob CP internados no Hospital de Clínicas de Porto Alegre (HCPA), para que dados de acompanhamento dessa população sejam relatados e contribuam para melhorar a qualidade de vida desses pacientes. O objetivo secundário foi descrever as características demográficas e clinicas e o desfecho clínico dos pacientes.

\section{MÉTODOS}

\section{Instrumento de Coleta de Dados}

Para a coleta de dados foi utilizado um instrumento padronizado de coleta de dados (Apêndice 1). As informações foram obtidas por meio de prontuários on-line no sistema AGH (Aplicativo de Gestão Hospitalar, implantado no ano 2000). Dados como sexo, idade, cidade de residência, internações anteriores, tratamentos realizados e duração da internação do paciente foram coletados a partir do cadastro do usuário no hospital. Outros dados como dieta, frequência respiratória, Escala de Morse (avalia risco de quedas - sendo risco baixo até 24 pontos na escala, moderado de 25 a 44, e alto igual ou maior de $45^{10}$ ), Escala de Braden (avalia risco de formação de úlceras de pressão - sendo risco médio de 15 a 18 pontos, moderado de 13 a 14, alto de 10 a 12, e altíssimo de 9 a $6^{11}$ ) e o escore de dor (escala de 1 a 10 - sendo dor leve de 1 a 3 , moderada de 4 a 6 , intensa de 7 a 9 , e insuportável é de $10^{12}$ ), assim como outros sintomas ou intercorrências, foram obtidos na evolução do paciente registrada diariamente também no prontuário on-line no sistema AGH. Os medicamentos utilizados durante a internação estudada foram coletados a partir da prescrição eletrônica. A coleta de dados foi realizada pelo pesquisador envolvido no projeto sob supervisão do farmacêutico co-orientador responsável, durante 2 meses.

\section{Amostra}

O presente trabalho foi um estudo exploratório para possibilitar o cálculo de tamanho amostral para o desenvolvimento de pesquisas posteriores, visto que não há dados suficientes para a determinação da amostra. Os dados existentes no atual momento não 
possibilitam cálculo estatístico de tamanho amostral para possíveis inferências que possam relacionar a utilização de medicamentos no $\mathrm{CP}$ e a qualidade de vida envolvida nesse desfecho. A diversidade cultural e as diferentes políticas públicas de saúde, assim como os diferentes sistemas de saúde internacionais, podem ocasionar diferenças no tratamento e no manejo do paciente sob CP; portanto, não seria adequada a utilização de dados internacionais para a determinação do tamanho amostral. O estudo objetivou determinar o perfil da população em estudo, bem como os medicamentos utilizados e os escores registrados pela equipe de enfermagem, para que se obtenham dados mais fiéis sobre essa população. Este estudo foi realizado com 30 pacientes, os quais foram selecionados aleatoriamente por conveniência, totalizando 34 internações (dois pacientes com duas internações e um com três internações no período analisado), ou seja, foram consideradas todas as internações (de cada um dos 30 pacientes) na unidade de cuidados paliativos (UCP).

\section{Delineamento}

O delineamento deste estudo foi transversal, retrospectivo, descritivo, com análise de prontuários eletrônicos de pacientes sob CP internados no HCPA, obtidos através do sistema informatizado AGH.

Foram incluídos no estudo pacientes internados no Núcleo de Cuidados Paliativos do HCPA. Foram excluídos do estudo indivíduos menores de 18 anos, pacientes que internaram no período anterior à implantação do Aplicativo de Gestão para Hospitais Universitários (AGHU) e pacientes que ainda não haviam recebido alta hospitalar.

\section{Análise de Dados}

Os dados obtidos a partir do instrumento padronizado de coleta de dados foram armazenados no programa Excel.

\section{Aspectos Éticos}

Este projeto foi aprovado pela Comissão Científica e Comissão de Pesquisa e Ética em Saúde, do Grupo de Pesquisa e Pós-Graduação (GPPG) do HCPA. Os pesquisadores participantes assinaram o Termo de Compromisso para Utilização de Dados Institucionais.

\section{RESULTADOS}

A UCP do HCPA foi implantada em 2007, conta com sete leitos e atende pacientes portadores de doença que ameace a vida, com o objetivo de promover alívio dos sintomas e melhorar a qualidade de vida dos pacientes ${ }^{6}$. No ano de realização deste estudo (2015), houve 24 internações em julho e 19 em agosto.
Foi encontrada uma média de 5,4 internações anteriores, independente da especialidade, entre os pacientes, com mínimo de uma internação e máximo de 15 internações no HCPA [mediana = 4 e desvio padrão $(\mathrm{DP})=3,71]$. A média de dias de internação no HCPA foi de 13,7 dias, sendo que a internação mais longa foi de 59 dias e a mais curta de 2 dias ( mediana $=6,5$ e DP $=15,26$ ). O número de dias de internação na UCP foi ligeiramente menor, com média de 7,0 dias, sendo que a internação mais longa foi de 23 dias e a mais curta de 1 dia (mediana $=5$, DP $=6,14$ ). Características demográficas dos pacientes encontram-se na Tabela 1, características clinicas na Tabela 2, características da dieta e da respiração e valores dos escores de Morse e de Braden na Tabela 3, e detalhamento do tratamento prévio na Tabela 4.

Dentre os pacientes pesquisados $(n=30)$, $6(20 \%)$ eram fumantes ativos, $13(43 \%)$ eram ex-tabagistas e 11 (37\%) eram não tabagistas. Três pacientes relataram dor diferente de zero nas escalas analisadas. Nos prontuários foram relatados fraqueza em 11 (32\%) pacientes e cansaço aos esforços em 3 (9\%) pacientes, enquanto que 20 (59\%) pacientes não apresentaram queixas. $O$ desfecho das internações encontra-se na Figura 1, sendo que $70 \%$ dos pacientes foi a óbito e os demais foram encaminhados para acompanhamento ambulatorial; destes, $6 \%$ foram encaminhados para o ambulatório sem especialidade especificada, $3 \%$ para equipe da dor, $18 \%$ para o setor de oncologia e $3 \%$ para o setor de pneumologia. Os medicamentos mais prescritos se encontram na Figura 2.

Tabela 1: Características demográficas da amostra $(n=30)$.

\begin{tabular}{lcc}
\hline & Valor bruto & $\%$ \\
\hline Gênero & & \\
$\quad$ Feminino & 16 & 53 \\
$\quad$ Masculino & 14 & 47 \\
Idade & & \\
$\quad<50$ & 2 & 7 \\
$50-59$ & 8 & 27 \\
$60-69$ & 7 & 23 \\
$70-79$ & 9 & 30 \\
$>80$ & 4 & 13 \\
Cor autodeclarada & & \\
Branca & 26 & 87 \\
Preta & 3 & 10 \\
Parda & 1 & 3 \\
Cidade de residência & & \\
Porto Alegre & 15 & 50 \\
Região metropolitana & 10 & 33 \\
Interior & 5 & 17 \\
\hline
\end{tabular}


Tabela 2: Características clinicas da amostra $(n=30)$.

\begin{tabular}{|c|c|c|}
\hline & Valor bruto & $\%$ \\
\hline \multicolumn{3}{|l|}{ IMC } \\
\hline Baixo & 22 & 73 \\
\hline Normal & 7 & 23 \\
\hline Sobrepeso & 0 & 0 \\
\hline Obesidade & 1 & 4 \\
\hline \multicolumn{3}{|l|}{ Tratamento prévio } \\
\hline Cirurgia & 13 & 43 \\
\hline Quimioterapia & 14 & 48 \\
\hline Radioterapia & 12 & 40 \\
\hline \multicolumn{3}{|l|}{ Motivo da internação ${ }^{1}$} \\
\hline CID A & 1 & 3 \\
\hline CID C & 20 & 59 \\
\hline CID E & 1 & 3 \\
\hline CID J & 5 & 14,5 \\
\hline CID K & 1 & 3 \\
\hline CID N & 1 & 3 \\
\hline CID R & 5 & 14,5 \\
\hline \multicolumn{3}{|l|}{ Neoplasia ${ }^{2}$} \\
\hline Esôfago & 5 & 18 \\
\hline Sistema respiratório & 5 & 18 \\
\hline Cavidade peritoneal & 1 & 3 \\
\hline Cólon/reto & 2 & 7 \\
\hline Estômago & 4 & 14 \\
\hline Leucemia & 1 & 3 \\
\hline Mama & 3 & 11 \\
\hline Melanoma & 2 & 7 \\
\hline Pâncreas & 1 & 3 \\
\hline Rim & 2 & 7 \\
\hline Útero & 2 & 7 \\
\hline
\end{tabular}

IMC - Índice de massa corporal; CID A - Algumas doenças infecciosas e parasitárias; CID C - Neoplasias; CID E - Doenças endócrinas, nutricionais e metabólicas; CID J - Doenças do aparelho respiratório; CID K - Doenças do aparelho digestivo; CID N - Doenças do aparelho geniturinário; CID R - Sintomas, sinais e achados anormais de exames clínicos e de laboratório, não classificados em outra parte. ${ }^{1} \mathrm{n}=34 .{ }^{2}$ um paciente estava internado por hepatite viral crônica.
Tabela 3: Características da dieta e da respiração e valores dos escores de Morse e de Braden da amostra $(n=34)$.

\begin{tabular}{lcc}
\hline & Valor bruto & $\%$ \\
\hline Dieta ${ }^{1}$ & & \\
VO & 21 & 62 \\
SNE & 6 & 18 \\
NPO & 7 & 20 \\
Respiração ${ }^{2}$ & & \\
E & 18 & 53 \\
D & 4 & 12 \\
D/E & 11 & 32 \\
E/D/B/T & 1 & 3 \\
Morse & & \\
Risco baixo & 0 & 0 \\
Risco médio & 11 & 32 \\
Risco alto & 23 & 68 \\
Braden & & \\
Risco médio ou baixo & 14 & 41 \\
$\quad$ Risco moderado & 8 & 24 \\
Risco alto & 10 & 29 \\
Risco altíssimo & 2 & 6 \\
\hline
\end{tabular}

${ }_{1}^{1}$ Dieta: VO - Via oral, SNE - Sonda nasoenteral, Npo - Nada por via oral. ${ }^{2}$ Respiração: E - Eupneico, D - Dispneico, B - Bradpneico, $\mathrm{T}$ - Taquipneico.

Tabela 4: Detalhamento do tratamento prévio.

\begin{tabular}{lc}
\hline \multicolumn{1}{c}{ Tratamento prévio } & Pacientes \\
\hline Quimioterapia + Cirurgia + & 2 \\
Radioterapia & 7 \\
Quimioterapia + Cirurgia & 1 \\
Quimioterapia + Radioterapia & 4 \\
Cirurgia + Radioterapia & 5 \\
Quimioterapia & 4 \\
Cirurgia & 1 \\
Radioterapia & 5 \\
Nenhum &
\end{tabular}

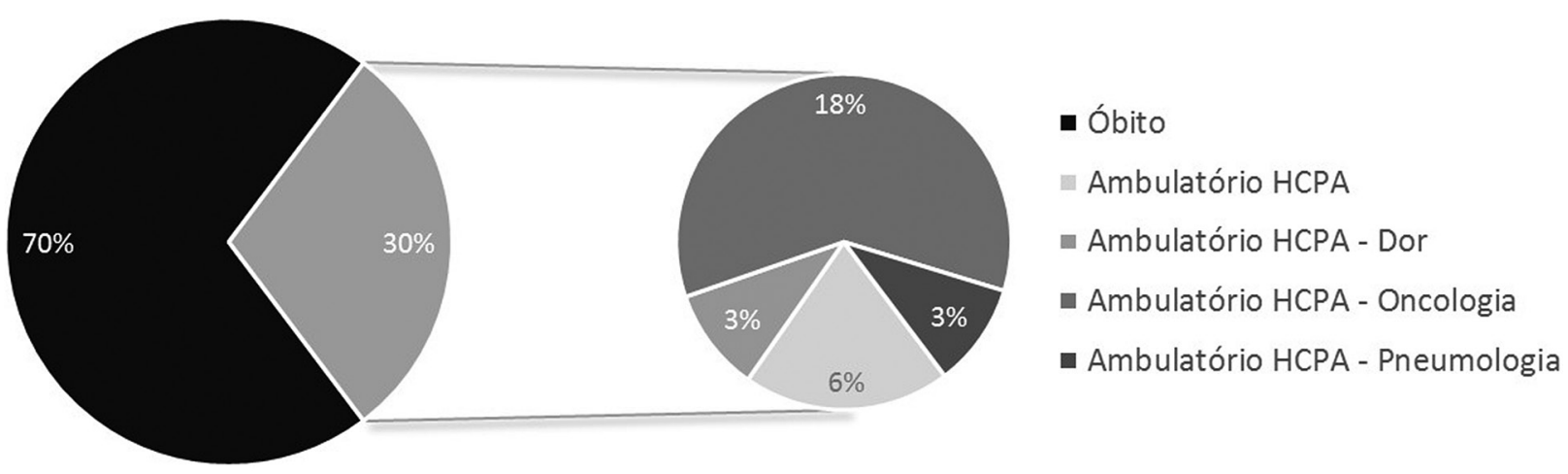

Figura 1: Desfecho das Internações. 


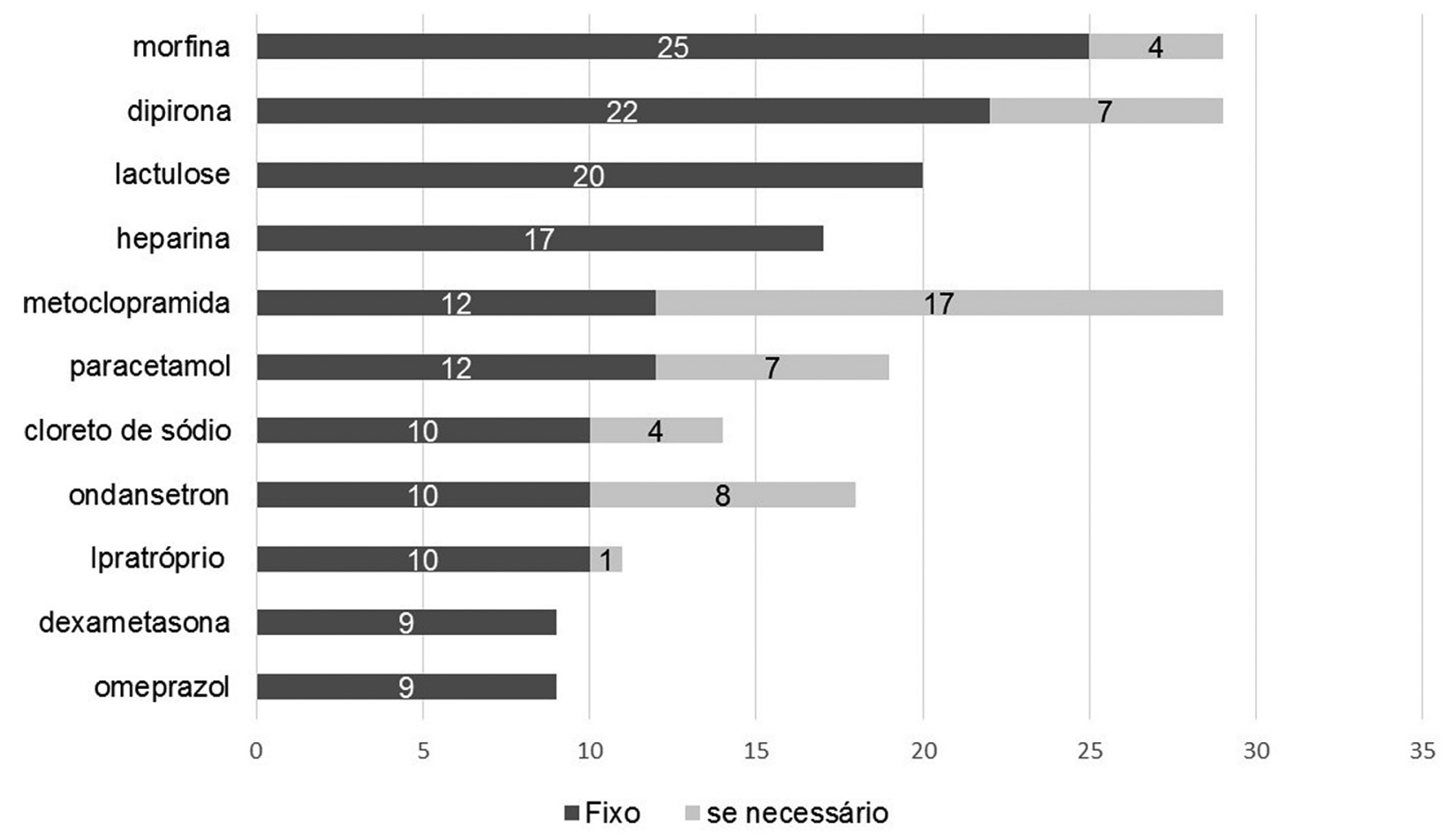

Figura 2: Medicamentos mais prescritos durante internação na UCP.

\section{DISCUSSÃO}

Agrande diversidade étnica e cultural de várias regiões do mundo deu início ao processo de miscigenação que constitui o atual povo gaúcho. Segundo o IBGE, em 2008 a população gaúcha era composta por $82,3 \%$ de brancos, $11,4 \%$ de pardos, $5,9 \%$ de negros e $0,4 \%$ de amarelos ou indígenas. Em 2010 a Região Sul do país possuía 27.386.891 habitantes; destes, $84,9 \%$ moravam em regiões urbanas e $15,1 \%$ em área rural. Quanto à proporção entre homens e mulheres na região, também em 2010 , havia $49,1 \%$ de homens e $50,9 \%$ de mulheres ${ }^{2}$. Todos esses dados podem ser comparados aos da Tabela 1.

A porcentagem de homens e mulheres internados na UCP do HCPA foi semelhante à da população geral do Rio Grande do Sul. A qualidade do atendimento primário também pode refletir nos dias de internação dos pacientes: pacientes da capital e região metropolitana eram mais propensos a aceitar a alta hospitalar, já os familiares de pacientes do interior possuíam maior receio de levar o paciente para casa, mesmo tendo uma maior dificuldade de acompanhá-lo no hospital, conforme relatos da equipe de saúde registrados nos prontuários desses pacientes.

A causa de internação hospitalar mais frequente entre os idosos no Brasil é a insuficiência cardíaca, $12,1 \%$ para as mulheres e $14,7 \%$ o para os homens.
Pneumonia, bronquite e acidente vascular cerebral também estão entre as principais causas de internação, tanto para homens quanto para mulheres ${ }^{3}$. O idoso consome mais os serviços de saúde, as internações hospitalares são mais frequentes e o tempo de ocupação do leito é maior, devido à multiplicidade de patologias ${ }^{3}$. No caso da amostra coletada, observou-se que as internações foram causadas tanto pelos motivos comuns da faixa etária citados acima como por neoplasia em estado avançado, o que pode ser observado nos diversos CID presentes na Tabela 2. Também nessa tabela observa-se uma maior prevalência de neoplasias de esôfago, sistema respiratório e estômago. Segundo Hallal et al., em um levantamento de dados históricos do Rio Grande do Sul, as localizações anatômicas mais frequentes nos tumores do sexo masculino foram pulmão, esôfago, próstata, estômago e cólon/reto; entre as mulheres foram mama, colo do útero/útero não especificado, pulmão, cólon/reto e estômago ${ }^{13}$.

Com relação ao tratamento prévio, há diversas combinações de tratamento entre cirurgia, quimioterapia e radioterapia, sendo que apenas dois pacientes foram submetidos aos três tratamentos ao longo da evolução da patologia, além de cinco pacientes que não passaram por nenhum tratamento (tabela 4). 
O tempo entre a descoberta da neoplasia, progressão natural da patologia e tratamento também leva ao índice de massa corporal reduzido encontrado na maioria dos pacientes, visto que aceleração de metabolismo, anorexia e caquexia neoplásicas são esperados em pacientes terminais ${ }^{14}$. Fadiga pode ser observada principalmente nesses pacientes mais debilitados.

Tabagismo entre os pacientes traz outro agravante à qualidade de vida: doença pulmonar obstrutiva crônica (DPOC). Mesmo com pequeno número amostral foi possível observar cinco pacientes com DPOC, três deles ex-tabagistas e dois fumantes ativos, mas não foi possível correlacionar estatisticamente esses valores com os dados respiratórios.

As prescrições analisadas para os 30 pacientes totalizaram 76 medicamentos diferentes; dentre estes, os mais prescritos encontram-se na Figura 2. Com a possibilidade de acúmulo de patologias crônicas, a polifarmácia é inevitável. A média de medicamentos prescritos para um paciente na UCP foi de cerca de 13 medicamentos por prescrição, sendo que a maior receita apresentou 25 medicamentos diferentes durante a internação, sem levar em consideração mudanças na posologia ou via de administração.

A avaliação do uso dos fármacos torna-se de grande importância para que se garanta a segurança do paciente em $\mathrm{CP}$ e o uso racional de medicamentos, ou seja, para que os pacientes recebam os medicamentos apropriados para suas condições clínicas, em doses adequadas às suas necessidades individuais, por um período adequado e ao menor custo para si e para a comunidade. O sucesso terapêutico depende dessa compreensão por parte do paciente e seus familiares. O farmacêutico se insere nesse cuidado quando realiza revisão de prescrição, capacitação da equipe de saúde e dos cuidadores, faz ajuste e conversão de dosagens e participa das equipes multidisciplinares ${ }^{15}$. Em estudo realizado por Hussainy et al. na Austrália, a inclusão de farmacêuticos nas equipes aumentou o conhecimento relacionado a medicamentos por parte da equipe de $\mathrm{CP}$ e dos pacientes, melhorando o manejo das prescrições e reduzindo erros relacionados ${ }^{16}$. Quando o paciente faz a escolha de receber $\mathrm{CP}$ no ambiente domiciliar, o reconhecimento da forma como essa terapia será manejada faz com que a alta hospitalar tenha êxito ou não ${ }^{17}$.

A realização de estudos na área de $\mathrm{CP}$ que avaliem a eficácia e segurança de medicamentos pode garantir uma melhor qualidade de atendimento, qualificando a unidade hospitalar ou o cuidado domiciliar. Reconhecer o momento em que o paciente necessita ficar internado ou ir para casa é fundamental para garantir o êxito do CP.

As limitações deste estudo podem ser atribuídas ao estudo ser retrospectivo, o que pode limitar a qualidade de análise dos dados em relação ao seu registro, pois não se consegue voltar ao caso para confirmar a veracidade.

A construção metodológica deste estudo possibilitou descrever o perfil da população, além de gerar conhecimento sobre os medicamentos utilizados na UCP e sobre variáveis demográficas e clínicas de pacientes internados nessa unidade. Conhecer o perfil de uso de medicamentos utilizados em uma UCP é um importante indicador de qualidade de assistência e saúde, possibilitando a assistência integral de forma efetiva e com atuação conjunta dos profissionais da equipe multidisciplinar.

\section{Agradecimentos}

À Equipe dos Cuidados Paliativos do Hospital de Clínicas de Porto Alegre e ao Programa de Pós-graduação em Assistência Farmacêutica - UFRGS pelo apoio acadêmico.

\section{REFERÊNCIAS}

1. Suzman R, Beard J. Global health and 3. Instituto Brasileiro de Geografia aging. Bethesda: National Institute of Aging; 2011 [citado em 2015 Out 30]. Disponível em: https://www.nia. nih.gov/research/publication/globalhealth-and-aging/preface

2. Instituto Brasileiro de Geografia e Estatística - IBGE. Indicadores sociais municipais. Brasília: IBGE; 2010 [citado em 2015 Out 31]. Disponível em: http://www.ibge. gov.br/home/estatistica/populacao/ censo2010/indicadores_sociais_ municipais/tabelas_pdf/tab1.pdf e Estatística - IBGE. Indicadores sociodemográficos e de saúde no Brasil. Brasília: IBGE; 2009 [acesso 2015 Out 30]. Disponível em: http:// biblioteca.ibge.gov.br/visualizacao/ livros/liv42597.pdf

4. Hermes HR, Lamarca IC. Palliative care: an approach based on the professional health categories. Cien Saude Colet. 2013;18(9):257788. http://dx.doi.org/10.1590/ S1413-81232013000900012. PMid:23989564.
5. Pan American Health Organization - PAHO. Health in the Americas, Country volume: Brazil. Washington: PAHO; 2012 [citado em 2015 Out 30]. Disponível em: http://www. paho.org/saludenlasamericas/index. php?option=com_docman\&task=doc view\&gid=118\&ltemid=

6. World Health Organization - WHO. Introduction. In: World Health Organization - WHO. Global atlas of palliative care at the end of life. $\mathrm{WHO}$; 2014. p. 4-9 [citado em 2015 Out 27]. Disponível em: http://www.thewhpca. 
org/resources/global-atlas-on-end-oflife-care

7. Clark D, Centeno C. Palliative care in Europe: an emerging approach to comparative analysis. Clin Med. 2006;6(2):197-201. http://dx.doi. org/10.7861/clinmedicine.6-2-197. PMid:16688982.

8. Blumhuber H, Kaasa S, De Conno F. The European Association for Palliative Care. J Pain Symptom Manage. 2002;24(2):124-7. http://dx.doi.org/10.1016/S08853924(02)00454-2. PMid:12231128.

9. Academia Nacional de Cuidados Paliativos - ANCP. O que são cuidados paliativos? São Paulo: ANCP; 2009 [citado em 2015 Out 26]. Disponível em: http://www.paliativo. org.br/ancp. php?p=oqueecuidados

10. Costa-Dias MJM, Ferreira PL, Oliveira AS. Adaptação cultural e linguística e validação da Escala de Quedas de Morse. Rev Enf Ref. 2014;IV(2):7-17. http://dx.doi.org/10.12707/RIII1382.
11. Cidral S, Silva WF, Visentin A, Borghi AC, Mantovani MF, Hey AP. Assessment of the risk of pressure ulcer development among hospitalized HIV/Aids patients. Rev Bras Enferm. 2016;69(1):96-101. http://dx.doi. org/10.1590/0034-7167.2016690113i. PMid:26871221.

12. Wells $N$, Pasero C, McCaffery M. Improving the quality of care through pain assessment and management. In: Hughes RG, editor. Patient safety and quality: an evidence-based handbook for nurses. Rockville: Agency for Healthcare Research and Quality; 2008. Chapter 17.

13. Hallal ALC, Gotlieb SLD, Latorre MRDO. Evolução da mortalidade por neoplasias malignas no Rio Grande do Sul, 1979-1995. Rev Bras Epidemiol. 2001;4(3):168-77. http://dx.doi.org/10.1590/S1415790X2001000300004.

14. Argilés JM, Busquets S, LópezSoriano FJ, Figueras M. Fisiopatología de la caquexia neoplásica. Nutr
Hosp. 2006;21(Suppl 3):4-9.

PMid:16768025.

15. Cortis LJ, McKinnon RA, Anderson C. Palliative care is everyone's business, including pharmacists. Am J Pharm Educ. 2013;77(2):21. http://dx.doi.org/10.5688/ajpe77221. PMid:23520403.

16. Hussainy SY, Box M, Scholes S. Piloting the role of a pharmacist in a community palliative care multidisciplinary team: an Australian experience. BMC Palliat Care. 2011;10(1):16. http://dx.doi org/10.1186/1472-684X-10-16 PMid:22035160.

17. Edwards SJ, Abbott R, Edwards J, LeBlanc M, Dranitsaris G, Donnan $\mathrm{J}$, et al. Outcomes assessment of a pharmacist-directed seamless care program in an ambulatory oncology clinic. J Pharm Pract. 2014;27(1):46-52. http://dx.doi. org/10.1177/0897190013504954. PMid:24065784.

Recebido: Dez 23, 2015 Aceito: Abr 21, 2016 
Apêndice 1: Instrumento Padronizado de Coleta de Dados.

Instrumento Padronizado de Coleta de Dados.

. Número Prontuário |

1. Data de nascimento:

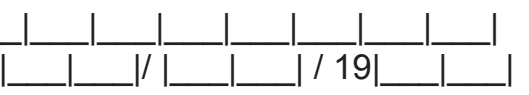

2. Sexo:

(1) Masculino (2) Feminino

3. Cor autodeclarada:

(1) Amarela (2) Branca (3) Parda

(4) Negra (5) Indígena

4. Peso:

5. Cidade de residência

6. Financiamento utilizado na internação

(1) Público (2) Privado Qual?

7. É a primeira internação do paciente?

(1) Sim (2) Não

8. Quantas internações anteriores o paciente teve?

9. Qual foi a duração das internações anteriores?

_______ dias

10. O paciente fez uso de algum medicamento antineoplásico nas internações anteriores?

(1) Sim (2) Não

11. O paciente fez uso de outro tratamento para o câncer?

(1) $\operatorname{Sim} \rightarrow$ (1.1) Cirurgia (1.2) Radioterapia (2) Não

12. Causa da internação hospitalar CID

13. Principal doença associada à internação hospitalar CID

14. Tabagismo

( ) Sim ( ) Não

15. Dados e escores do paciente

\begin{tabular}{|c|c|c|c|c|c|}
\hline Data & Dieta & Respiração & Morse & Braden & Dor \\
\hline & & & & & \\
\hline & & & & & \\
\hline & & & & & \\
\hline & & & & & \\
\hline & & & & & \\
\hline & & & & & \\
\hline & & & & & \\
\hline & & & & & \\
\hline
\end{tabular}


Preencher utilizando as seguintes siglas e dados:

Dieta: VO - Via oral

SNE - Sonda nasoenteral

NPO - Nada por via oral

Respiração: E - Eupneico

D - Dispneico

B - Bradpneico

$\mathrm{T}$ - Taquipneico

Morse: Escore a partir de 0

Braden: Escala a partir de 0

Dor: Escala de 0 a 10

16. O paciente apresentou
( ) Náuseas
( ) Vômitos
( ) Diarreia
( ) Constipação

17. Houve relato de fadiga?

() Sim () Não

18. Quais medicamentos o paciente utilizou durante a internação?

\begin{tabular}{|l|l|l|l|l|l|}
\hline Medicamento & Dose $(\mathbf{m g})$ & Posologia & Via de administração & Início & Fim \\
\hline & & & & & \\
\hline & & & & & \\
\hline & & & & & \\
\hline & & & & & \\
\hline
\end{tabular}

19. Quantos dias o paciente ficou internado na unidade?

।_____ dias

20. Qual a condição do paciente na alta da unidade?

(1) Alta melhorando

(2) Óbito

(3) Outra. Qual?

21. Qual o encaminhamento pós-alta hospitalar?

(1) Ambulatório HCPA

(2) Médico externo ao HCPA

(3) Outro hospital em Porto Alegre

(4) Hospital em outra cidade

(5) Outro Qual?

22. Houve alguma intercorrência durante a internação? Qual? 